\title{
La réalité soviétique au prisme du regard des femmes polonaises (1930-1939)
}

Soviet Reality in Polish Women's Gaze (1930-1939)

\section{Agata Zawiszewska}

Traducteur : Agnieszka Grudzińska

\section{OpenEdition \\ Journals}

Édition électronique

URL : https://journals.openedition.org/recherchestravaux/3405

DOI : 10.4000/recherchestravaux.3405

ISSN : 1969-6434

Éditeur

UGA Éditions/Université Grenoble Alpes

\section{Édition imprimée}

ISBN : 978-2-37747-301-4

ISSN : 0151-1874

Référence électronique

Agata Zawiszewska, «La réalité soviétique au prisme du regard des femmes polonaises

(1930-1939)», Recherches \& Travaux [En ligne], 98 | 2021, mis en ligne le 28 juin 2021, consulté le 09

août 2021. URL : http://journals.openedition.org/recherchestravaux/3405 ; DOI : https://doi.org/

10.4000/recherchestravaux.3405

Ce document a été généré automatiquement le 9 août 2021.

(c) Recherches \& Travaux 


\title{
La réalité soviétique au prisme du regard des femmes polonaises (1930-1939)
}

\author{
Soviet Reality in Polish Women's Gaze (1930-1939)
}

Agata Zawiszewska

Traduction : Agnieszka Grudzińska

1 Tout au long de l'entre-deux-guerres polonais, le reportage demeure un "genre négocié » - pour reprendre la formule d'Urszula Glensk ${ }^{1}-$, tout juste en train de conquérir son autonomie générique. Il est au cœur d'un large débat sur les lignes de partage entre différentes formes de discours et sur la répartition des compétences entre artistes, journalistes, scientifiques, hommes politiques, etc. Dans ce débat, réel comme symbolique, les voix masculines dominent nettement mais, pour la première fois dans l'histoire de la culture polonaise, de nombreuses femmes se font également entendre. Car les femmes sont désormais à l'origine de conceptions et de pratiques nouvelles et en plein essor. Tout particulièrement, les femmes s'illustrent dans la prose documentaire dont on peut considérer qu'elle constitue une forme de proto-reportage. En effet, les femmes ont été les premières à s'infiltrer au cœur des quartiers misérables, à démasquer les violences, aussi bien celles commises hors-la-loi comme celles pratiquées par les institutions officielles. Les affinités entre les femmes et le reportage, et en particulier le reportage naissant, ont déjà été relevées par Zygmunt ZiĄtek, pour qui la participation des femmes a contribué à « aiguiser la dimension émotionnelle du projet de dévoilement propre au reportage ${ }^{2}$ ». Hanna Kirchner a, quant à elle, souligné que les évolutions de la littérature polonaise au lendemain de 1918 favorisaient les femmes: «la poétique du quotidien, la découverte d'un exotisme du quotidien, l'autobiographisme, les formes documentaires ${ }^{3} »$. Les autrices en question contribuent également à redéfinir les contours de la figure auctoriale: elles revendiquent une écriture d'intervention, motivée par un contexte ou un événement précis, plus proche du journalisme que de l'idéal de l'accomplissement esthétique. Elles écrivent souvent en marge d'une activité professionnelle, dans la presse, pour le bulletin d'une société de 
géographie, pour répondre à la demande d'une institution sociale, dans une brochure féministe ou encore pour inciter d'autres femmes à suivre leurs traces. Elles se montrent particulièrement attentives à la vie quotidienne, à la mode vestimentaire, au cadre de vie et, naturellement, à la place et à la condition des femmes.

2 Les textes analysés ci-dessous peuvent être qualifiés de proto-reportages. Ils témoignent à la fois d'une pratique aux limites encore incertaines et de l'appropriation de cette pratique par les femmes. Le corpus est constitué des textes suivants, écrits par des femmes, à l'occasion de voyages en URSS, dans les années 1930 (présentés dans l'ordre de leur apparition dans l'étude) :

- Moja wyprawa na Pamiry w r. 1929 [Mon expédition dans le Pamir en 1929] de Jadwiga ToeplitzMrozowska (rédigé en italien et publié à Rome en 1930, puis traduit vers le polonais et publié en 1934) ;

- Sowieckie państwo pracy. Wrażenia z podróży inspektora pracy [Le monde du travail en Union soviétique. Impressions de voyage d'un inspecteur du travail] (19355) de Janina Miedzińska (première publication dans le quotidien Gazeta Polska [Le Quotidien polonais], 1934) ;

- Dymy nad Azją [Les Fumées au-dessus de l'Asie] (19346) de Wanda Kragen ;

- ZSRR w oczach kobiety [L'URSS vue par une femme] (19367) de Halina Lenczewska-Borman ;

- Listy z Nowego Wschodu [Lettres du nouvel Est] (1935') de Stefania Zahorska (première publication dans l'hebdomadaire Wiadomości Literackie [Les Nouvelles littéraires], 1935).

\section{Contexte}

3 Les récits étudiés doivent tout d'abord être replacés dans leur contexte. En Europe comme en Amérique, on écrit alors beaucoup sur la Russie d'après 1917. Il faut également prendre en compte la situation politique, économique et culturelle spécifique à l'Europe après la Grande Guerre ${ }^{9}$. Le contexte politique se caractérise par l'instabilité de l'ordre fondé sur le traité de Versailles, la peur croissante face à la montée du nazisme à partir de 1933 et les changements au sein du pouvoir soviétique qu'Andrzej Walicki ${ }^{10}$ a décrits comme le passage progressif du communisme totalitaire de Lénine au totalitarisme communiste de Staline. Le contexte économique, c'est la crise, le développement de l'industrie de l'armement en Allemagne ainsi que l'industrialisation, l'urbanisation et la modernisation de l'URSS. Le contexte culturel, enfin, est marqué par le sentiment d'une crise de la culture occidentale et une dégradation de l'image de l'intellectuel européen qui s'était cristallisée au moment de l'affaire Dreyfus ${ }^{11}$.

4 La tradition des voyages littéraires et des récits de voyage est alors très vivace, favorisée par l'essor du tourisme - à la fois symptôme et symbole de la modernité -, qui fait écho aux fantasmes impérialistes des classes moyennes occidentales. Tous ces éléments permettent de comprendre pourquoi l'Europe capitaliste et démocratique observe avec grand intérêt la Russie postrévolutionnaire, à la fois attraction touristique et point de départ d'un retour réflexif sur soi. Les intellectuels constituent une part importante des voyageurs qui se rendent en URSS. Après 1918, ils ont à choisir entre deux postures : celle du révolutionnaire professionnel et celle du clerc ${ }^{12}$. S'engager de façon active dans la politique afin de changer le monde ou bien se consacrer entièrement à une œuvre dénuée de toute ambition d'influencer la réalité : tel est le choix qui se pose aux intellectuels et artistes européens et polonais. Parmi ces derniers, le débat s'intensifie à partir de 1927, année qui voit la parution du livre de Julien Benda, 
La Trahison des clercs $^{13}$. Le dilemme est particulièrement sensible dans les textes des intellectuels progressistes, socialistes ou sympathisants socialistes. En effet, les années 1914-1918 ont sensiblement affaibli leur autorité et mis au jour l'ambiguïté de leurs positions, entre idéal universaliste et attachement à l'état nation ${ }^{14}$.

Dans ce contexte, les récits de voyage en URSS rédigés par des intellectuels progressistes sont souvent l'objet de larges débats dans les pays européens. En effet, ils contiennent non seulement des observations et des diagnostics concernant l'équilibre des forces en présence dans l'entre-deux-guerres mais encouragent également une réflexion sur le statut social de l'intellectuel moderne. Une partie de ces derniers adopte la conviction de l'effondrement de l'Occident démocratique, libéral et rationaliste (confortée après 1918 par les écrits d'Oswald Spengler, Arnold Toynbee, Karl Jaspers, José Ortega y Gasset, Aldous Huxley, Julien Banda et, en Pologne, Florian Znaniecki). D'autres mettent leurs espoirs dans le communisme, perçu comme un antidote au fascisme italien, au national-socialisme allemand et à l'intensification du nationalisme dans d'autres pays (ces espoirs s'expriment chez Herbert George Wells, George Bernard Shaw, Bertrand Rusell, Panaït Istrati, Romain Rolland, André Gide, André Malraux, Lion Feuchtwanger, Egon Erwin Kisch, Malcolm Muggeridge, Arthur Koestler et, en Pologne, Władysław Broniewski ou Antoni Słonimski). Les voix critiques envers l'Union Soviétique, comme celles de Gide, d'Istrati ou de Słonimski en Pologne, sont certes écoutées mais souvent appréhendées avec une distance critique parce que - comme nous l'avons dit plus haut - l'Union Soviétique constitue pour beaucoup la seule alternative au nazisme et à la montée des nationalismes mais aussi parce que le discours prosoviétique reproduit le discours colonial propre aux pays occidentaux ${ }^{15}$. En effet, les territoires de l'Eurasie russe sont à l'Union Soviétique ce que le Far West, l'Afrique ou l'Amérique du Sud sont aux puissances occidentales: des contrées "sauvages", "arriérées", "non civilisées", où il faut porter les valeurs, les mécanismes et les institutions fondamentales de la culture occidentale ${ }^{16}$.

On peut diviser les voyageurs se rendant en URSS en trois groupes: les professionnels (les scientifiques et les ingénieurs venus en URSS dans le cadre d'un contrat de travail), les "pèlerins révolutionnaires" et les touristes ${ }^{17}$. Chaque groupe obéit à une motivation différente : travail, foi ou curiosité. Chaque motivation, enfin, se traduit à sa manière dans le discours sur la Russie, et chaque discours crée une image différente de l'Autre. Les femmes, certes moins nombreuses que les hommes, figurent aussi parmi les chercheurs, ingénieurs, pèlerins et touristes qui déferlent en Union Soviétique par vagues successives. La proportion des voyageuses est cependant moindre pour plusieurs raisons évidentes : le manque de confort, les prétendus dangers guettant les femmes occidentales dans la "Russie sauvage ", la conviction encore tenace à l'époque selon laquelle il n'est pas convenable pour une femme de voyager non accompagnée. Les femmes célèbres voyagent d'ailleurs souvent en compagnie d'hommes: la journaliste Geneviève Tabouis accompagne Édouard Herriot ; Beatrice Webb, son époux Sidney; Lady Astor, son mari, Lord Astor et George Bernard Shaw. Les femmes qui voyagent seules obéissent aux mêmes motivations que les hommes - professionnelles, idéologiques et touristiques -, mais leur cas étant marginal, le voyage devient de surcroît un signe d'émancipation ${ }^{18}$. En effet, l'émancipation féminine constitue ${ }^{19}$ le centre de gravité et l'originalité des récits de voyage féminins en URSS dans les années 1930. Nous allons le montrer grâce à quelques exemples, tout en posant la question de la vision de l'Autre véhiculée par ces récits de femmes. 


\section{Le discours scientifique au féminin}

Mon expédition dans le Pamir en 1929 de Jadwiga Toeplitz-Mrozowska est publié en 1929. L'autrice, originaire d'une famille de propriétaires terriens, s'est lancée dans une carrière de comédienne contre l'avis de sa famille ${ }^{20}$. Après $1907^{21}$, année de la fin de sa carrière, elle séjourne à l'étranger et rencontre Józef Toeplitz, directeur adjoint de la Banca Commerciale Italiana à Milann ${ }^{22}$. Elle l'épouse en 1918 et s'installe définitivement à Milan, où elle anime la vie culturelle de l'élite milanaise grâce à un salon musical et s'emploie à promouvoir la culture polonaise. Elle aménage les jardins de sa villa de Varèse, où elle cultive des plantes rapportées de ses nombreux voyages ${ }^{23}$.

8 En 1919, Mrozowska commence à voyager vers l'Est. En 1930, elle a déjà visité l'Inde, Ceylan, la Birmanie, l'Iran, le Tibet et le Cachemire. Durant son voyage dans le Pamir, qu'elle considère comme sa plus grande réussite, elle explore et décrit la région frontalière du Tadjikistan, de l'Afghanistan et de la Chine. Elle parvient à expliquer les changements cycliques du niveau des eaux du lac Zorkul et à ouvrir de nouvelles voies dans les montagnes du Pamir. Pour la remercier de sa contribution à la science, la Société géographique italienne donne son nom au col qu'elle a découvert: Passo J. Toeplitz-Mrozowska (4 $200 \mathrm{~m}$ au-dessus du niveau de la mer) et lui décerne sa médaille d'or, qu'elle attribue ainsi pour la première fois à une femme ${ }^{24}$. Mrozowska raconte ses voyages lors de conférences publiques, dans des articles, des bulletins géographiques et dans des livres rédigés en italien ${ }^{25}$. C'est une des premières femmes polonaises à faire du voyage un véritable style de vie, ce qui est rendu possible grâce à la fortune de son mari, aux acquis du mouvement féministe du début du $\mathrm{xx}^{\mathrm{e}}$ siècle et à la longue tradition italienne du tourisme ${ }^{26}$. En se remémorant ses voyages à la fin de sa vie elle écrira : «[...] ce n'était pas seulement de l'ambition, mais l'impétueux élan d'un être humain assoiffé de vie primitive et de liberté27 ${ }^{2}$.

Mon expédition dans le Pamir en 1929 se présente sous la forme du compte-rendu d'expédition scientifique sur le terrain. L'introduction, de la plume de Kazimiera Jeżowa, célèbre géographe polonaise, enseignante et travailleuse sociale, fait le récit de dix années de travail sur le terrain mené par Mrozowska. Le texte de Mrozowska lui-même abonde en informations détaillées sur le relief, les conditions climatiques, la faune et la flore, la toponymie, l'aspect et les mœurs des habitants. Les tournures passives y sont nombreuses; le recours au conditionnel pour formuler des hypothèses, fréquent; le vocabulaire spécialisé abonde et le récit est étayé par de nombreuses données chiffrées; l'expression d'émotions est évitée. Le livre comprend une carte et des photographies. Enfin, l'expédition a également été immortalisée sur $2000 \mathrm{~m}$ de pellicule, donnant ainsi naissance au premier film sur le Pamir.

10 Force est cependant de constater que l'autrice se fonde essentiellement sur des données déjà fournies par des voyageurs occidentaux qui l'ont précédée et non sur les savoirs propres à la population locale auxquels elle ne prête pas attention. Elle énumère les beautés et les richesses du Tadjikistan, du Kirghizistan et de l'Afghanistan mais c'est toujours dans la perspective d'une colonisation et d'une exploitation. Elle emploie systématiquement les mots comme «local » ou « indigène ». Elle perçoit les Tadjiks et les Kirghizes uniquement au prisme des valeurs capitalistes, individualistes, libérales et démocratiques, comme le travail, l'esprit de conquête, l'égalité des sexes, ce qui la 
conduit à reproduire l'image, nettement dévalorisante, du « sauvage fainéant », friand de pacotille :

Tout le travail est effectué par les femmes, parce que le Kirghize estime, depuis des siècles, que le travail déshonore l'homme libre et il y a lieu de penser qu'il va rester fidèle à cette conception confortable de l'honneur pendant des siècles encore. Bien évidemment, tout ce qu'on reçoit des Kirghizes doit être payé non en monnaie mais en produits manufacturés. Les tissus de coton blancs, noirs et à fleurs sont très prisés de même que les couteaux, les allumettes et les montres. Les femmes sont comblées par les miroirs, le savon, le parfum et les perles de verre ainsi que par tout cosmétique pouvant colorer leur visage. Elles adorent les poudres blanches, bleues, les crayons marron et les rouges à lèvres. (p. 71-72)

11 Les tigres, tortues et lézards ne sont pas uniquement des objets de contemplation esthétique mais aussi des trophées de chasse. L'or, le fer, le sel et les pierres précieuses ne doivent pas rester inutilisés et Mrozowska regrette que leur exploitation présente des difficultés: "l'exploitation de ces richesses aujourd'hui encore se heurte à des difficultés quasiment insurmontables, dues à l'absence de voies de communication " (p. 46). Elle approuve entièrement les actions entreprises par le pouvoir soviétique au Tadjikistan: la garantie de la liberté de pratiquer l'islam, la construction d'écoles et d'hôpitaux, l'approvisionnement en nourriture, et, en même temps, l'encouragement à adopter le modèle coopératif.

12 Alors que dans les récits occidentaux, les intellectuels voyageant en URSS se comparent aux intellectuels soviétiques, Toeplitz-Mrozowska, voyageuse polonaise, se mesure à la femme autochtone, femme passive qui attend son mari et mène une vie sédentaire. Elle oppose à ce modèle sa propre image de femme occidentale active, dynamique, engagée dans le monde. En fin de compte, le discours de cette femme émancipée est un discours de type colonial ${ }^{28}$.

\section{Le discours expert au féminin}

Le Monde du travail en Union soviétique de Janina Miedzińska est un exemple de discours d'expert assumé par une femme : c'est le discours d'une professionnelle au sujet d'un domaine qui constitue sa spécialité. Plusieurs femmes occidentales se sont illustrées dans ce type de récit: Jessica Smith, Alice Withrow Field, Esther Conus, Elli Winter ${ }^{29}$. Toutes ces autrices travaillaient pour des institutions sociales et des organisations non gouvernementales chargées de la protection et de la santé des femmes et des enfants. Elles se sont rendues en URSS à la fin des années 1920 et au début des années 1930 pour y observer les avancées en matière d'égalité des sexes, de la protection des femmes et de leurs conditions de travail. Leurs récits, qui s'adressent aux soviétologues et aux féministes des pays occidentaux, se veulent objectifs mais en tant que féministes professionnelles et observatrices internationales, elles adoptent une posture de supériorité vis-à-vis des femmes soviétiques ${ }^{30}$. La traduction de ces travaux vers le polonais et, un peu plus tard, l'écriture de ce genre d'ouvrages par des Polonaises, s'inscrivent dans le contexte d'un intérêt généralisé pour la Russie de la part des Polonais. En effet, les relations diplomatiques entre la Pologne et l'URSS sont en plein réchauffement dans les années $1930^{31}$. Le discours expert des femmes sur la Russie est accueilli avec intérêt, mais les auteurs des recensions sur ces écrits avouent y apprécier davantage la critique de l'URSS que l'idée même d'émancipation. 
14 Miedzińska est une pionnière dans le domaine des écrits concernant l'impact du travail sur la santé physique et mentale des ouvriers, qu'elle publie dans la revue polonaise Praca i Opieka Społeczna [Travail et aide sociale] ainsi que dans les brochures financées par le Ministère de la Protection Sociale ${ }^{32}$. Inspectrice du travail d'obédience marxiste, elle se rend en URSS en tant que représentante de l'État polonais. Conformément aux dogmes du socialisme et aux conceptions d'August Bebel, elle considère que la lutte des classes une fois terminée, celle des sexes le sera automatiquement. Dans ses récits, le statut des femmes-ouvrières constitue une des questions abordées et non pas l'objet principal de l'analyse ${ }^{33}$.

Le Monde du travail en Union soviétique se présente comme une étude scientifique objective, documentée, comportant des statistiques, des extraits d'interviews et de nombreuses citations de la presse locale. Miedzińska évite systématiquement toute prise de position idéologique univoque sur les conditions de travail des femmes soviétiques dans les usines. Dans l'introduction de son ouvrage, elle précise que son livre est le produit d'un voyage organisé par l'Institut des Affaires sociales [Instytut Spraw Społecznych], dans le but de " prendre connaissance des méthodes de protection du travail afin de s'en inspirer éventuellement chez nous » (p. 5). C'est pourquoi « elle n'a pas de caractère scientifique et ne prétend pas à l'exhaustivité » (p. 6). Miedzińska peine à garder une position pleinement objective et à se contenter de recueillir les faits. En tant qu'inspectrice du travail et socialiste, elle s'attendait à voir en union soviétique "sinon une réalisation complète des aspirations du monde du travail, du moins une politique sociale qui aurait le bien du travailleur pour unique objectif » (p. 7). Pourtant, c'est justement sa bonne connaissance de Marx qui lui permet de dissiper l'illusion d'un paradis socialiste et de voir, dans les usines soviétiques, la réalisation d'un taylorisme poussé à l'extrême. L'individu y est une bête de somme; le seul objectif visé reste l'augmentation du rendement au mépris de la santé des ouvriers; les femmes sont assignées à des tâches considérées dans les pays capitalistes comme fatales pour leur fertilité. Il faut savoir que Miedzińska, comme tout le mouvement féministe polonais, exige l'égalité des droits en même temps qu'une protection accrue pour les femmes, en raison de leurs spécificités physiologiques. Elle ne peut admettre qu'en URSS, les hommes et les femmes aient les mêmes droits mais aussi les mêmes devoirs (p. 75). Le projet initial de Miedzińska était de recueillir et systématiser des faits en se gardant de tout projet de synthèse. Cependant la découverte de statistiques sur les accouchements et sur les maladies des femmes et des enfants en totale contradiction avec les études menées dans des usines occidentales depuis la moitié du XIX siècle, finit par donner au récit de Miedzińska une tonalité décidément négative.

\section{Le discours touristique au féminin}

Les livres de Wanda Kragen [Les Fumées au-dessus de l'Asie] et de Halina LenczewskaBorman [L'URSS vue par une femme] sont des exemples de récits touristiques féminins. Les deux récits ont plusieurs traits en commun. Tout d'abord, les deux autrices sont des touristes modernes qui participent à un voyage en URSS organisé par l'agence Intourist. Elles apprécient le confort et la présence d'un guide tout au long de cette aventure organisée ; elles considèrent la Russie comme une attraction touristique et la visitent avec leurs yeux. La préférence donnée au sens visuel signifie que l'attention est concentrée sur la surface du réel, sur la perception du monde tel qu'il se présente au 
premier et unique coup d'œil. Cependant, les deux autrices ne se rendent pas en URSS dans un but exclusivement touristique. Leurs voyages sont aussi des «voyages sentimentaux ». Wanda Kragen se rend de Moscou jusqu'en Sibérie pour retrouver un ancien amant. Halina Lenczewska-Borman cherche à Moscou les souvenirs du temps où elle y allait à l'école, puis visite une vieille amie à Toula. Une mélancolie, une quête de soi-même, de celle qu'on a été autrefois, traverse les deux livres. La clé du passé est détenue par des personnes incarnant un ensemble d'expériences et d'émotions qu'on nomme habituellement "premier amour» et "amitié féminine». Enfin, les deux autrices accordent beaucoup d'attention à la réglementation des voyages en tramway et en chemin de fer ${ }^{34}$ ainsi qu'aux descriptions de l'aspect extérieur des habitants de l'URSS ${ }^{35}$.

Le récit de Kragen, plus que celui de Lenczewska-Borman, est régi par les règles discursives du guide touristique. Il propose force informations pratiques à l'intention des touristes à venir : l'équipement nécessaire, les démarches à la douane, la sociabilité pendant les trajets en train de plusieurs jours, l'aménagement des chambres d'hôtel, le menu des restaurants dans les hôtels et en ville, le fonctionnement des bons alimentaires, les taux de change, le prix des courses en taxi et des billets de tram, les itinéraires des cars d'Intourist, les conditions de visite des musées de la Révolution, du mausolée de Lénine, des théâtres de Meyerhold et de Vakhtangov, des kolkhozes exemplaires, etc. L'autrice décrit tout cela sans commentaires, elle ne manifeste aucune ambition de regarder dans les coulisses de la réalité soviétique, ne pose pas aux habitants de questions pièges concernant leur opinion sur la révolution. Elle se concentre sur ce que Moscou et l'URSS veulent bien lui montrer, et non pas sur ce qui est caché aux regards des visiteurs. Chaque particularité soviétique est, pour Kragen, une simple spécificité locale, un objet exposé dans le grand musée du monde, une attraction touristique stricto sensu. C'est pourquoi ses reportages sur le voyage en train ou en tramway, ainsi que ceux de Lenczewska-Borman et de Zahorska, peuvent être considérés comme les premiers exemples en Pologne d'études anthropologiques sur les espaces d'anonymat que Marc Augé appelle «les non-lieux ${ }^{36} »$. Elles décrivent des micro-événements comme l'achat d'un ticket de tram, elles sont attentives aux conversations, aux attitudes, à la manière dont les corps occupent l'espace. Mais la touriste polonaise veut goûter les charmes de Moscou conformément aux standards et aux scénarios européens, telle par exemple la satisfaction immédiate des demandes des clients. Chez Kragen, le récit rappelle alors les descriptions orientalisantes des « indigènes paresseux »:

À Moscou personne n'est pressé. Surtout les serveurs. Il y en a beaucoup, on ignore pour quelle raison, ils sont très polis et bien élevés mais ils se déplacent à la vitesse d'une mouche dans du sirop. Quand tu appelles un serveur, il répond " tout de suite » mais il s'approche de ta table seulement un bon quart d'heure plus tard. Une demi-heure plus tard, après une série de nouvelles urgences, il t'apporte enfin ton plat, qui n'est pas toujours celui que tu as commandé. (p. 19-20)

Il en est de même lors des descriptions du comportement de la foule dans les rues. Alors que les habits portés dans les centres et à la périphérie des villes occidentales signalent et en même temps confirment et pérennisent les différences ethniques, de classe et de sexe entre les habitants, les habits arborés par les Moscovites abolissent ces différences. Tout le monde se ressemble et tous ressemblent aux couches les plus pauvres de la population. Le centre de la capitale donne l'impression que « de vieux vêtements étaient venus ici de toute l'Europe, comme si Moscou tout entier s'habillait 
chez des chiffonniers » (p. 31). Les chapeaux, manteaux ou fourrures à l'apparence plus européenne signalent l'appartenance à l'élite, ils sont les attributs de «l'étranger, du diplomate, de l'ingénieur ou du spécialiste étranger » (p. 32).

En lisant Les Fumées au-dessus de l'Asie, il est difficile d'établir quelle est la véritable opinion de l'autrice sur ce qu'elle a vu en URSS. À la fin de son récit elle affirme : «Et me voilà à nouveau à Varsovie. Je ne suis pas sûre d'avoir bien compris ce que j'ai vu de l'autre côté. Je ne sais qu'une chose [...] Là-bas, c'est différent. » Il y a cependant à la fin de son livre, un fragment qui trahit les sentiments de l'autrice, justement parce qu'il révèle le véritable motif de son voyage. Au moment de quitter Kouznietsk, en Sibérie, et de faire ses adieux à un ami de jeunesse perdu de vue pendant la révolution (p. 168), elle fond en larmes: «Lorsque mon ami me serre dans ses bras pour la dernière fois, lorsque je sens sur mes lèvres son baiser, les larmes longtemps retenues jaillissent de mes yeux et ma bouche peine à retenir ce cri suivant: "Reviens! Fuis ce pays !" " (p. 236).

Le récit de Borman née Lenczewska, femme d'Antoni Borman, éditeur des Wiadomości Literackie [Les nouvelles littéraires], la revue littéraire la plus importante de l'entre-deuxguerres polonais, est différent. Cette journaliste, collaboratrice de plusieurs magazines féminins, rédactrice, à partir de 1939, de la revue Kobiety $w$ świecie $i w$ domu [Les Femmes dans le monde et à la maison], femme du monde élégante bien connue des salons de Varsovie, propose un récit de voyage en URSS saturé de commentaires sur les habits, les accessoires, les coiffures et le maquillage. L'URSS vue par une femme - pour reprendre le titre de son ouvrage - est avant tout un pays où le système de la mode occidentale est absent ${ }^{37}$, ce qui, pour la styliste professionnelle, est synonyme de barbarie esthétique à portée idéologique. Cette barbarie n'est pas uniquement due à l'absence de codes vestimentaires lisibles, résultant d'une pénurie de matières premières et des insuffisances de la technologie, ce qui serait encore acceptable aux yeux de la voyageuse. Elle découle directement du système soviétique qui, en abolissant - du moins en prétendant abolir - les différences de classe, abolit d'autres différences et avant tout celle entre les sexes : « La foule est curieusement uniforme. Tout le monde se ressemble. Comment les couples peuvent-ils se former dans cette situation? Comment font-ils pour savoir que c'est lui, que c'est elle, alors qu'ils sont tous semblables?» (p.18) Le théâtre de la vie quotidienne où, comme le montre Erving Goffman, les hommes et les femmes se livrent à - ou contemplent la - «parade des genres $^{38}$ » et de la différence, peut avoir lieu uniquement dans le cadre d'une économie capitaliste, qui est pour Lenczewska-Borman la base de la culture occidentale.

21 Les deux principales observations de l'autrice sont l'omniprésence des femmes en URSS, en particulier dans les métiers considérés en Europe (et donc en Pologne) comme "masculins" (chauffeur, conducteur, gardien, contrôleur) et leurs tenues vestimentaires, adaptées au climat et au travail exercé au lieu d'être régies par le statut social, l'âge ou le sexe. Les femmes sont partout: "La première chose qui saute aux yeux c'est la femme à tous les postes possibles » (p. 7) ; « sur les murs on était en train de coller des affiches. Bien sûr, ce sont des femmes qui les collent. À l'intersection des voies de tramways, c'est une femme qui opère. [...] Le conducteur est, bien sûr, une femme.» (p. 25); "À l'angle de la rue Petrovka une femme-policière régule la circulation. Une femme-gardienne arrose la rue avec de l'eau. [...] Une femmechauffeur conduit une voiture remplie de militaires.»(p. 26). Les descriptions des vêtements deviennent, sous la plume de Lenczewska-Borman, une expression du 
sentiment de supériorité de la femme occidentale par rapport à la femme soviétique. Le système de la mode occidentale en constitue la pierre de touche et l'infériorité de l'Autre est construite méthodiquement au moyen d'oppositions binaires entre les étrangers habillés à l'européenne et les habitants de l'URSS. La polarisation conduit à l'identification de «l'ordre européen" à la culture et du "chaos soviétique " à l'absence de la culture ${ }^{39}$ : «Les femmes ont toutes l'air d'institutrices de campagne, elles portent des robes en toile blanche, toutes simples, ridicules, presque toujours blanches. »(p. 17). Ou encore :

La fille est débraillée, des mèches de cheveux dépassent de dessous son foulard, le tricot aux manches remontées et la jupe ne sont pas propres. Sur ses genoux, elle tient une valise et dans les bras, un lapin vivant. Même dans la réalité bolchevique, la vision est extraordinaire. [...] Elle connaît très bien la pièce. C'est la quinzième fois qu'elle y assiste. Elle obtient des billets gratuits à l'usine. Elle va très souvent au théâtre, surtout celui de Meyerhold, parce que c'est cette orientation qui lui convient le mieux. J'ai failli tomber de ma chaise - cette orientation lui convient très bien. Pourtant elle a l'air d'une cuisinière. Ce visage obtus! (p. 45-46)

Les tenues abolissent la différence entre les sexes:

Presque toutes se promènent tête nue. Parfois un béret à l'arrière de la tête, bien que ce soit plutôt une tenue masculine. Parfois un chapeau de paille masculin - ce sont en général de vieilles dames. (p. 18)

Souvent ces descriptions vestimentaires se superposent à des descriptions des corps des femmes russes, qui rappellent les portraits anthropologiques des "sauvages", qui les renvoient à la sphère de la nature et de la reproduction :

Les femmes sont curieusement mal faites. De petite taille. Les hanches larges et les poitrines volumineuses témoignent $\mathrm{du}$ fait qu'elles constituent la première génération de femmes à avoir compris que le corps n'est pas uniquement fait pour la reproduction et le travail. (p. 17)

Le sentiment de supériorité esthétique éprouvé par Lenczewska-Borman est lié à sa conviction que les femmes occidentales en URSS sont investies d'une mission civilisatrice qui consiste à détruire la communauté révolutionnaire sans classes et sans différence des sexes, grâce à l'introduction de la mode et de la décoration moderne des intérieurs. Cette mission incombe non seulement à celles qui voyagent, mais surtout aux femmes occidentales installées en URSS, épouses des chercheurs, ingénieurs ou médecins, qui doivent être des modèles à imiter par la communauté locale. C'est le cas de l'amie de jeunesse de l'autrice, une femme qui a étudié à Paris, connue pour sa beauté et son élégance, qui a tourné la tête de plus d'un homme. Installée à Toula, elle ne peut plus briller par son intelligence et sa beauté, provoquer la jalousie par ses tenues, séduire. Cependant elle s'efforce de faire en sorte que son couple, son appartement et son investissement social soient pour les « locales » un exemple du sens des responsabilités, de l'ardeur au travail et des capacités organisationnelles, propres à la culture occidentale.

En fin de compte, contrairement aux affirmations de Lenczewska-Borman selon lesquelles six semaines en URSS "c'est assez pour voir des choses et d'autres, mais décidément trop peu pour tirer des conclusions » (p. 235), le regard porté sur les femmes témoigne de la désapprobation profonde de l'autrice à l'égard de l'ordre soviétique. 


\section{Le discours esthétique au féminin}

Les Lettres du Nouvel Est de Stefania Zahorska est un exemple de discours esthétique au féminin. Socialiste, journaliste, historienne de l'art, philosophe et écrivaine ${ }^{40}$, Zahorska s'est rendue en URSS pour étudier la réalisation cinématographique auprès de Sergueï Eisenstein. Dans son récit, elle adopte le point de vue de la professionnelle de l'architecture, de la peinture et du cinéma. Elle contemple la réalité soviétique à travers le dogme du modernisme architectural, du futurisme et de l'expressionnisme. Le modernisme, le futurisme et l'expressionnisme constituent les outils les plus adéquats pour analyser et interpréter la modernitée ${ }^{41}$. Le langage de l'art, pour Zahorska, permet de rendre compte du nouvel ordre social sans en déformer la vérité ${ }^{42}$. Pourtant Zahorska finit par formuler un jugement sur l'URSS aussi négatif que celui de Kragen, qui fait appel au discours touristique, ou que celui de Lenczewska-Borman qui recourt au discours de la mode :

Ça se passait toujours ainsi pour moi à Moscou. Les premières sensations visuelles blessaient l'œil, tombaient dans la pupille comme une poignée de gravats, dure, informe, un manque d'organisation, de formes, de proportions, il fallait que j'apprenne à fermer les yeux. (p. 124)

Toutes ces questions sont posées d'emblée. Le premier texte, intitulé «Je prends le tramway à Moscou » témoigne d'une appréhension négative par Zahorska de tout ce qui est soviétique. Il contient même la plupart des stéréotypes négatifs sur l'Autre colonisé. Les sens de l'autrice perçoivent les odeurs, les images, les sons " inesthétiques", produits par les " autochtones » sales, ivres et bruyants, aux traits grossiers. Elle éprouve de la répulsion face aux «murs dénudés d'un grand bâtiment jaune et aux trous dans le pavage où s'accumule de la boue automnale ", face aux « cochers en chapeau d'astrakan, sales, imprégnés d'humidité et de l'odeur de rennes moisies» (p. 79), face aux "visages larges aux traits épais» (p. 81) recouverts de "casquettes fripées", de "foulards gris », de "chapkas du temps des tsars » ou de "vareuses usées d'avant la révolution" (p. 81). L'autrice reconnait ouvertement que, pour elle, ce sont les villes comme Paris et Londres qui servent de référence pour estimer le niveau de civilisation d'une ville. Elle avoue qu'elle a peur de l'Est mais son désir de "connaître la culture prolétarienne est plus fort et prend le dessus sur les réflexes d'autodéfense » (p.87).

Cependant le jugement le plus radical sur l'URSS est placé dans la bouche d'une étrangère installée dans le pays depuis longtemps, épouse d'un ingénieur qui y travaille. Elles font penser aux paroles de Kurtz à la fin d'Au cœur des ténèbres de Joseph Conrad :

[...] une vieille dame se tient assise là et demande en français: "Vous-êtes une étrangère? Ça se voit tout de suite d'après vos vêtements... qu'est-ce que je suis heureuse de pouvoir parler à une étrangère. Mon mari est ingénieur. Nous sommes venus avant la guerre..." Elle se penche vers moi : "C'est dur, très dur... Ce ne sont pas des conditions pour les gens bien... J'ai grandi dans une bonne famille. Et là, c'est la sauvagerie ! La sauvagerie..." (p. 91)

La vision selon laquelle les habitants de l'URSS sont des barbares puisqu'ils ne connaissent pas le langage de l'art occidental, est soulignée par des descriptions expressionnistes de l'architecture et des intérieurs. Quant au peuple russe, Zahorska le voit comme une masse passive façonnée par l'Histoire, dénué de goût et d'individualité. Tout cela lui inspire dégoût, angoisse, sentiment de supériorité, et justifie une distance 
qu'il faut nécessairement garder, même si elle comprend la difficulté à vivre entre « la misère de la toile cirée verte et l'ignominie de l'assiette ébréchée. » (p. 80)

Pour l'autrice, les canons de la beauté sont fixés par les architectes du Bauhaus alors qu'en Russie, où on pourrait pourtant s'attendre à trouver le modernisme dans toute sa splendeur, il y en a peu et même quand il existe, il est « misérable et bancal » (p. 103), «apporté de l'extérieur [...] mal nettoyé, inachevé et incongru» (p. 124). En revanche «le néoclassicisme avance victorieux, alors même qu'un savant soviétique y voyait le style de l'impérialisme » (p. 103). Des maisons laides sont occupées par des gens laids dénués de goût qui se complaisent dans le kitsch des « canapés en velours avec dossier haut et accoudoir en bois ", des "nénuphars art-déco fleurissant sur les sofas", des " petits vases en verre de toutes les couleurs pleins de roses et d'œillets en papier ", des «tableaux représentant des cascades assourdissantes et des jeunes filles» (p. 97). Observant la foule en admiration devant les bibelots kitsch en vente dans un Univermag (grand magasin d'État), Zahorska ne peut s'empêcher d'y voir une manifestation de l'aspiration contrerévolutionnaire des prolétaires au confort des « intérieurs bourgeois » et des "maisons closes». Elle refuse d'y voir une forme de nostalgie du peuple amputé de son histoire. Selon Zahorska, le péché capital des femmes russes est leur penchant pour le kitsch et le luxe ostentatoire. En effet, conformément aux représentations dominantes en URSS comme ailleurs, les femmes soviétiques sont rendues responsables de la sphère privée, en particulier au moment du deuxième plan quinquennal, qui mise sur l'industrie légère, le textile et l'amélioration du cadre de la vie ordinaire.

Parmi les récits étudiés ci-dessus, celui de Zahorska, la seule intellectuelle stricto sensu parmi les autrices dont il a été question, porte sur les femmes soviétiques le jugement le plus sévère et le plus méprisant. Aucune femme n'est traitée avec sérieux ou respect. Les interlocuteurs qu'elle considère comme ses égaux sont tous des hommes éduqués : médecin, directeur d'un kolkhoze, bibliothécaire, camarades de l'école de cinéma, et avant tout Eisenstein. Même un ouvrier ivre, avec qui elle entame une conversation dans un tramway, s'avère être un interlocuteur plus intéressant que sa logeuse, qu'elle décrit en exagérant ses propos et gestes, comme s'il s'agissait d'un personnage de film comique. Les descriptions expressionnistes de la «kolkhozienne-femelle», de la «kolkhozienne-animal», de la «kolkhozienne-sauvage» sont sans conteste la représentation la plus condescendante des femmes soviétiques qu'on puisse trouver dans les récits des voyageuses polonaises en URSS :

Je n'ai pas tout de suite compris ce qui se passait. Quelques voix féminines ont commencé à piailler à toute vitesse, caquetant comme au poulailler, le caquetage augmentait, s'intensifiait, il y avait beaucoup de poules, de plus en plus [...] leurs bras tendus battaient comme des ailes, les femmes se levaient de leurs chaises, rouges, indignées, leur cri montait, toute la salle hurlait, prise de convulsions. (p. 133)

Au milieu de la pièce une masse compacte tournoyait, semblable à de la pâte à gâteau mi-liquide. Les femmes qui dansaient agitaient leurs bras au-dessus de leurs têtes, elles faisaient des pas invisibles dans l'obscurité de leurs jupes. Larges, lourdes et tremblantes, elles se frottaient les unes aux autres telles des vaches aveugles. Leurs faces rouges, riantes, inondées de sueur montaient, les foulards arrachés des têtes tournoyaient dans les airs, les seins, gros comme des citrouilles, se balançaient sous les tuniques rapiécées, les jupons gonflaient sur les derrières rebondis. (p. 138-139) 


\section{Le regard d'une Polonaise sur l'URSS}

31 Les récits de voyage en URSS dans les années 1930 laissés par les autrices polonaises sont habituellement relégués à la marge des études sur le sujet ou alors traités dans les notes de bas de page. Pourtant leur lecture ne met pas uniquement en évidence les différences entre les regards masculin et féminin sur la réalité soviétique, ou les liens entre le regard et la différence de classe. Elle dévoile aussi les ressemblances frappantes de ces récits de femmes sur le plan de l'idéologie: bien que Toeplitz-Mrozowska, Miedzińska, Kragen, Lenczewska-Borman et Zahorska ne s'expriment pas en tant qu'intellectuelles et qu'elles se gardent d'émettre des jugements politiques univoques sur l'ordre postrévolutionnaire, elles font preuve d'une forte réticence à son égard, tout comme la majorité des hommes polonais. Dans les récits féminins, cette antipathie se cristallise autour de la question de l'émancipation, qu'on a pris l'habitude, dans les années 1920, d'appeler « la question féminine ».

Les écrits de femmes que nous avons étudiés ont pour point commun leur constat de l'impossible émancipation des femmes en URSS. La conviction y est très forte de la supériorité de la femme moderne occidentale sur la femme soviétique et du féminisme occidental sur sa variante soviétique. La nécessité d'introduire en Russie les acquis du mouvement féministe européen y est présentée comme une « mission civilisatrice » qui doit être menée par les femmes occidentales. Elle doit s'effectuer dans la sphère privée, parallèlement à la mission menée par les hommes occidentaux dans le domaine de la science et de la technologie. Ainsi, le féminisme véhicule un rapport à l'Autre rempli de présupposés universalistes, voire impérialistes, évoquant l'attitude de l'Occident face aux colonies ${ }^{43}$. Les Polonaises construisent leur supériorité sur les citoyennes soviétiques en se plaçant en position d'autorité en tant qu'exploratrices, chercheuses, observatrices de l'égalité des sexes, du tourisme ou du style. Elles décrivent la femme soviétique sans aucune considération comme une masse indifférenciée, insuffisamment civilisée ou bien trop primitive: négligée, démodée, surmenée, mal-éduquée, dépourvue d'identité culturelle, d'indépendance et de satisfaction érotique, se complaisant dans le kitsch, bruyante comme un enfant ou un animal. Dans ces récits, la femme soviétique ne s'exprime jamais en son nom propre, car on ne la laisse pas parler - elle est l'Autre muette. Et même si elle parvient à se frayer un chemin à travers le flot de paroles narcissiques des autrices polonaises, ses propos sont déformés et accueillis au mieux avec incrédulité, au pire avec une suspicion de mauvaise foi. La femme soviétique, inférieure, pauvre et sédentaire, devient un objet de conquête idéologique, qu'il faut protéger, contrôler et prendre par la main. Ainsi les écrits des voyageuses polonaises nous en apprennent plus sur elles-mêmes que sur les femmes soviétiques.

\section{NOTES}

1. U. Glensk, Historia słabych. Reportaż $i$ życie $w$ dwudziestoleciu miĘdzywojennym (1918-1939) [Histoire des faibles. Le reportage et la vie dans l'entre-deux-guerres], Cracovie, Universitas, 2014, p. 78. 
2. Z. ZiĄtek, Wiek dokumentu. Inspiracje dokumentarne $w$ polskiej prozie wspótczesnej [Le siècle du document. Les inspirations documentaires dans la prose polonaise contemporaine], Varsovie, Wydawnictwo IBL PAN, 1999, p. 83.

3. H. Kirchner, « Pisarki miĘdzywojennego dwudziestolecia » [« Les écrivaines de l'entre-deuxguerres »], A.Żarnowska, A. Szwarc (dir.), Równe prawa $i$ nierówne szanse. Kobiety $w$ Polsce miĘdzywojennej [Égalité des droits et inégalité des chances. Les femmes dans la Pologne de l'entre-deuxguerres], Varsovie, DiG, 2000, p. 249. Voir aussi A. Araszkiewicz, Zapomniana rewolucja. Rozkwit kobiecego pisania $w$ dwudziestoleciu miĘdzywojennym [La révolution oubliée. L'essor de l'écriture des femmes dans l'entre-deux-guerres], Varsovie, Wydawnictwo IBL PAN, 2014.

4. J. Toeplitz-Mrozowska, Moja wyprawa na Pamiry w r. 1929, traduit de l'italien par K. Jeżowa, Lvov-Varsovie, KsiĄżnica Atlas, 1934, série « Dookoła Ziemi » [ « Autour de la terre »], (original : La Prima spedizione italiana attraverso i Pamiri 1929, Rome, Società Geografica Italiana, 1930). Le récit de Jadwiga Toeplitz-Mrozowska, bien qu'écrit en italien puis traduit vers le polonais, peut être considéré comme un ouvrage polonais suivant la catégorisation de Jerzy Koch qui distingue l'emprunt (zapożyczenie) et le transfert (przeniesienie). Koch utilise les concepts forgés par le linguiste néerlandais Frans Van Coetsem dans Loan Phonology and the Two Transfer Types in Language Contact, Dordrecht, Foris, 1988. Cf. Jerzy Koch, "Zapożyczenie" ( borrowing) a "przeniesienie" (imposition) w strukturze badań recepcyjnych. Postulat badawczy i jego egzemplifikacja na przykładzie recepcji literatury niderlandzkiej na poczĄtku 20 wieku [L'emprunt (borrowing) et le transfert (imposition) dans la structure des études de réception : l'exemple de la réception de la littérature néerlandaise au début du $\mathrm{XX}^{e}$ siècle] dans Agata Zawiszewska et Aneta Borkowska, Nie tylko Zachód. Recepcja literatur obcych $w$ czasopismach polskich 20 wieku [Il n'y a pas que l'Occident. La réception des littératures étrangères dans les revues polonaises au $\mathrm{XX}^{e}$ s.], Łask-Szczecin-Toruń, Leksem, 2007.

5. J. Miedzińska, Sowieckie państwo pracy. Wrażenia z podróży inspektora pracy [Le monde du travail en Union soviétique. Impressions de voyage d'un inspecteur du travail], Varsovie, Instytut Wydawniczy, «Biblioteka Polska », 1935.

6. W. Kragen, Dymy nad AzjĄ, Varsovie, Tow. Wydawnicze, « Rój », 1934.

7. H. Lenczewska-Bormanowa, ZSRR w oczach kobiety, Varsovie, Tow. Wydawnicze « Rój », 1936.

8. S. Zahorska, Listy z Nowego Wschodu, in Wybór pism. Reportaże, publicystyka, eseje [Textes choisis. Reportages, articles, essais], A. Nasiłowska (éd.), Varsovie, IBL, 2010.

9. E. Pogonowska, Czytanie Nowej Rosji. Polskie spotkania ze Związkiem Radzieckim w latach trzydziestych 20 wieku [Lire la Russie Nouvelle. Les rencontres polonaises avec l'URSS dans les années 1930], Lublin, UMCS, 2012.

10. C. Walicki Andrzej, « Od totalitarnego komunizmu do komunistycznego totalitaryzmu » [« Du communisme totalitaire au totalitarisme communiste »], Marksizm i skok do królestwa wolności. Dzieje komunistycznej utopii [La Marxisme et le saut dans le royaume de la liberté. Histoire de l'utopie communiste], Varsovie, PWN, 1996.

11. Sur l'affaire Dreyfus vue de Pologne: E. Bieńkowsk, Spór odziedzictwo europejskie. MiĘdzy świętym i świeckim [Le conflit autour de l'héritage européen. Entre le sacré et le profane], Varsovie, W.A.B., 1998.

12. R. Aron, Le Spectateur engagé. Entretiens avec Jean-Louis Missika et Dominique Wolton [1981], Paris, Éditions de Fallois, 2004.

13. J. Benda, La Trahison des clercs [1927], Paris, Grasset, coll. « Les Cahiers rouges », 2003.

14. W. T. Starr, Romain Rolland. One against all. A Biography, La Haye/Paris, Mouton, 1971.

15. M. Heller et A. Nekrich, L'Utopie au pouvoir. Histoire de l'URSS, traduit du russe par W. Berelowitch, Paris, Calmann-Lévy, 1982.

16. E. Saïd, L'Orientalisme : l'Orient crée par l'Occident, trad. C. Malamoud, Paris, Le Seuil, 1980 ; Culture et impérialisme, trad. P. Chemla, Fayard/Le Monde diplomatique, 2000. Voir aussi E. Kuźma, 
Mit Orientu $i$ kultury Zachodu $w$ literaturze XIX $i$ XX wieku [Le mythe de l'Orient et les cultures occidentales dans la littérature des XIX ${ }^{e}$ et XX $X^{e}$ siècles], Szczecin, Wydawnictwo Naukowe WSP, 1980.

17. S. R. Margulies, The Pilgrimage to Russia. The Soviet Union and the Treatment of Foreigners 1924-1937, University of Wisconsin Press, Madison, 1968 ; P. Hollander, Political Pilgrims. Travels of Western Intellectuals to the Soviet Union, China and Cuba (1928-1978), Oxford University Press, New York, 1981.

18. H. Dietlind, «Kobieta w podróży. Czas emancypacji, czas wolności czy kontynuacja codzienności?» [«La femme en voyage. Émancipation, liberté ou toujours le même quotidien?»], dans A. Żarnowska et A. Szwarc (dir.), Kobieta i kultura czasu wolnego [La femme et la culture du temps libre], Varsovie, DiG, 2001 ; M. Olkuśnik, « Kobieta w podróży na przełomie 19 i 20 wieku. MiĘdzy próbą emancypacji a presją "podwójnej moralności” » [ « La femme en voyage à la fin du XIX $X^{e}$ et au début du XX $x^{e}$ siècle. Entre tentative d'émancipation et pression d'une "double morale" »], dans A. Żarnowska et A. Szwarc (dir.), Kobieta i małżeństwo. Społeczno-kulturowe aspekty seksualności. Wiek 19 i 20 [La femme et le mariage. Aspects sociaux-culturels de la sexualité. XIX et $\mathrm{XX}^{e}$ siècles], Varsovie, DiG, 2004.

19. D. Kałwa, Kobieta aktywna w Polsce miedzywojennej. Dylematy środowisk kobiecych [La femme active dans la Pologne de l'entre-deux-guerres. Dilemmes des milieux féminins], Cracovie, Historia Jagiellonica, 2001.

20. B. Czajecka, $Z$ domu w szeroki świat. Droga kobiet do niezależności w zaborze austriackim w latach 1890-1914 [De la maison vers le vaste monde. Le chemin des femmes vers l'indépendance dans la partie autrichienne de la Pologne dans les années 1890-1914], Cracovie, Universitas, 1990, p. 197-202.

21. Entrée « Toeplitz-Mrozowska Jadwiga », dans Zbigniew Wilski (dir.), Słownik biograficzny teatru polskiego [Dictionnaire biographique du théâtre polonais], vol.2, 1900-1994, Varsovie, PWN, 1994, p. 476-478; Ł. Iwanczewska, «Jadwiga Toeplitz-Mrozowska. Pozostać sob Ą... » [ "Jadwiga Toeplitz-Mrozowska. Rester soi-même... »], dans Krakowski szlak kobiet. Przewodniczka po Krakowie emancypantek [L'itinéraire cracovien des femmes. Une guide du Cracovie des femmes émancipées], E. Furgał (éd.), Cracovie, Fondation Espace des femmes, 2009.

22. L. Toeplitz, Il banchiere, al tempo in cui nacque, crebbe, e fiorì la Banca Commerciale Italiana [Le banquier à l'époque de la naissance, du développement et de la prospérité de la Banca Commerciale Italiana], Milan, Milono Nuova, 1963 ; I. Diomede, Banca, finanza e industria in Italia. In una corrispondenza tra Bonaldo Stringher e Giuseppe Toeplitz (1919-1930) [Banque, finance et industrie en Italie. Correspondance entre Bonaldo Stringher et Giuseppe Toeplitz (1919-1930)], Soveria Mannelli, Rubbettino, 2005.

23. K. T. Toeplitz, «Józef i Ludwik - "włoscy Toeplitzowie” [«Joseph et Louis, "les Toeplitz italiens"»], Rodzina Toeplitzów. KsiĄżka mojego ojca [La Famille Toeplitz. Le livre de mon père], Varsovie, Iskry, 2004.

24. Entrée "Toeplitz-Mrozowska Jadwiga », dans W. Słabczyński, Słownik podróżników polskich [Dictionnaire des voyageurs polonais], Varsovie, Wiedza Powszechna, 1992, p. 312.

25. Cf. E. Toeplitz-Mrozowska, Visioni orientali [Visions orientales], Milan, Mondadori, 1930 ; Sine ira. Nel Paese dell'URSS. Rifflesioni di chi ha vissuto sotto il regime zarista e viaggiato nel paese dell'URSS [En URSS. Réflexions de ceux qui ont vécu sous le régime tsariste et ont voyagé en URSS], Milan, Mondadori, 1933. Elle a publié en polonais ses souvenirs sous le titre Słoneczne życie [Une vie pleine de soleil], Cracovie, Wydawnictwo Literackie, 1963.

26. G. Alù, Italian women's Mezzogiorno, in Beyond the Traveller's Gaze. Expatriate Ladies Writing in Sicily (1848-1910), New York/Oxford, Peter Lang, 2008.

27. J. Toeplitz-Mrozowska, Une vie pleine de soleil, ouvr. cit., p. 270.

28. M. L. Pratt, Imperial eyes. Studies in Travel Writing and Transculturation, London/New York, Routledge, 1992. 
29. J. Smith, Woman in Soviet Russia, NY, Vanguard Press, 1928 ; A. Withrow Field, The Protection of Women and Children in Soviet Russia, London, E.P. Dutton and Co., 1932; M. Koniũs Ėsfir \& V. Mikhailovna Fediãevskaiã, The Protection of Motherhood and Childhood in the Soviet Union, Moscou/Leningrad, State Medical Editorship, 1933 ; W. Elli, Red Virtue. Human Relationships in the New Russia, Londres, Harcourt Brace and Co., 1933 ; G. N. Serebrennikov, The Position of Women in the USRR, Londres, Victor Gollancz 1937.

30. C. Talpade Mohanty, "Under Western Eyes. Feminist scholarship and colonial discourse ", dans P. Williams \& L. Chrisman (éd.), Colonial Discourse and Postcolonial Theory. A Reader, New York, Columbia University Press, 1994.

31. Il ne faut pas oublier qu'avant de retrouver son indépendance en 1918 la Pologne a été, depuis la fin du XVIII ${ }^{\mathrm{e}}$ siècle, partagée entre trois puissances voisines : la Russie, la Prusse et l'Autriche (puis Autriche-Hongrie). De 1919 à 1921 une guerre, ayant pour enjeu des territoires situés à la limite des deux États, a opposé la toute jeune Pologne et la non moins jeune Union Soviétique. Il est intéressant de remarquer que la Pologne est aussi, d'une certaine manière un pays longtemps colonisé par l'Empire russe.

32. Cf. J. Miedzińska, "Nauka zawodu” w przemyśle irzemiośle [L'apprentissage du métier dans l'industrie et l'artisanat], Varsovie, Główna Drukarnia Wojskowa, 1931; Polityka państwa wobec młodzieży pracującej 1930-1933 [La politique de l'État face aux jeunes travailleurs. 1930-1033], Varsovie, Min. Opieki Społecznej, 1933 ; Na niemieckim froncie pracy [Sur le front allemand du travail], Varsovie, Biblioteka Polska, 1937 ; «Praca kobiet w rozlewniach wódek. Przyczynek do badań wysiłku przy pracy " ["Le travail des femmes dans les usines de mise en bouteilles de vodka. Contribution à l'analyse de l'effort physique au travail »], Praca i Opieka Spoleczna [Le travail et l'aide sociale],1938, $\mathrm{n}^{\circ}$ 2, p. 139-157.

33. J. Miedzińska, The Protection of Women Workers and Minores in Poland. Report of the Chief Inspector of Woman Labour in Poland, trad. du polonais par M. Bamforth, Londres, Polish Women Committee for International Collaboration, 1941 ; Zagadnienia ochrony pracy w Polsce [Questions de la protection de travail en Pologne], Londres, Szkoła Nauk Politycznych i Społecznych, 1961; Polityka socjalna (prawo pracy) [La politique sociale (le droit du travail)] Londres, Instytut Badania Zagadnień Krajowych, 1965.

34. T. Wojciech, Ikona nowoczesności. Kolej w literaturze polskiej [Une icône de la modernité. Le chemin de fer dans la littérature polonaise], Wrocław, Wydawnictwo Uniwersytetu Wrocławskiego, 2007.

35. E. Pogonowska, «Semiotyka ubioru mieszkańców czerwonej Rosji na podstawie polskich relacji podróżniczych z lat trzydziestych 20 wieku » [ «La sémiotique vestimentaire en Russie soviétique d'après les récits de voyage polonais des années 1930 »], Napis, XXIV, 2018.

36. Marc Augé, Non-lieux, introduction à une anthropologie de la surmodernité, Paris, Le Seuil, 1992.

37. J'utilise ce terme conformément à son interprétation structuraliste proposée par Roland Barthes dans Système de la mode, Paris, Le Seuil, 1967.

38. E. Goffman, «La ritualisation de la féminité », Actes de la recherche en sciences sociales, vol. 14, 1977, p. 34-50.

39. Trinh T. Minh-ha, Woman, Native, Other. Writing Postcoloniality and Feminism, Bloomington, Indiana University Press, 1989.

40. M. Elżbieta Cybulska, Potwierdzone istnienie. Archiwum Stefanii Zahorskiej [L'existence attestée. Les archives de Stefania Zahorska], Londres, Polska Fundacja Kulturalna, 1988.

41. S. Zahorska, S. Frenkiel (éd.), SzczĘŚliwe oczy. Wybór studiów i esejów z dziedziny filozofii, historii $i$ krytyki sztuk plastycznych lat 1921-1960 [Les yeux heureux. Études philosophiques, historiques et critiques sur les arts plastiques des années 1921-1960], Londres, Polska Fundacja Kulturalna, 1970 ; Szkice o literaturze i sztuce, [Esquisses sur la littérature et l'art], P. KĄdziela (éd.), Varsovie, Twój Styl, 1995.

42. A. Pilch, Symbolika form i kolorów. O krytyce artystycznej Stefanii Zahorskiej [La symbolique des formes et des couleurs. La critique de l'art selon Stefania Zahorska], Cracovie, KsiEgarnia Akademicka, 2004. 
43. Cf. L. Gandhi, Postcolonialism and Feminism, in Postcolonial Theory. A Critical Introduction, Sydney, Allen\&Unwin, 1998; B. Pat, Woman and Empire. Representations in the Writings of British India (1858-1900), New Delhi Orient, Orient Black Swann, 2002.

\section{RÉSUMÉS}

Le texte propose une lecture critique des récits de voyage en URSS écrits par des Polonaises et publiés dans les années 1930. L'analyse se propose de montrer les spécificités de ces récits. Les outils analytiques mis en œuvre sont empruntés aux études de genre et aux études postcoloniales. On peut ainsi observer comment le discours émancipateur se mêle au discours patriarcal colonial, sur trois plans : le statut de la femme européenne émancipée et son point de vue impérialiste, l'image de la femme soviétique présentée comme une "sauvage " et l'identification des critères d'émancipation à la mission civilisatrice occidentale en URSS.

The paper presents a critical reading of travel accounts of journeys to USSR written by Polish women and published in Poland in the 1930s. The analysis shows similarities and differences between those travel accounts. Methodological tools used to that end come from the field of gender and postcolonial studies. This approach reveals the entanglement of the emancipation discourse with the patriarchal colonial discourse on three different levels: the status of the European emancipated woman as an imperialist, the picture of the "Russian woman" as a "savage" and the identification of emancipation criteria with the Western civilization mission in the USSR.

\section{INDEX}

Mots-clés : reportage polonais, 1918-1939, écritures féminines polonaises, récit de voyage, URSS Keywords : Polish reportage, 1918-1939, Polish women's writing, reportage, travel story, USRR

\section{AUTEURS}

\section{AGATA ZAWISZEWSKA}

Université de Szczecin

Agata Zawiszewska est professeur à l'Université de Szczecin. Elle se spécialise dans la recherche sur les revues socio-culturelles et la littérature féminine de l'entre-deux-guerres en Pologne. Elle a notamment publié : Recepcja literatury rosyjskiej na łamach Wiadomości Literackich. 1924-1939 [La Réception de la littérature russe dans la revue Wiadomości Literackie. 1924-1939], éditions de l'Université de Szczecin, 2005 ; Zachód w oczach liberałów. Literatura niemiecka, francuska i angielska na łamach Wiadomości Literackich. 1924-1939 [L'Occident vu par les libéraux. La littérature allemande, française et anglaise dans Wiadomości Literackie. 1924-1939], éditions de l'Université de Szczecin, 2006 ; Życie świadome. O nowoczesnej prozie intelektualnej Ireny Krzywickiej [Une vie consciente. La prose intellectuelle moderne d'Irena Krzywicka], éditions de l'Université de Szczecin, 2010 ; MiĘdzy MłodA PolskĄ, Skamandrem i AwangardĄ. Kobiety piszĄce wiersze na poczĄtku 20 wieku [Entre la Jeune Pologne, 
le groupe Skamander et l'avant-garde. Les poétesses du début du $\mathrm{XX}^{e}$ siècle], éditions de l'Université de Szczecin, 2015 ; Ster pod redakcją Pauliny Kuczalskiej-Reinschmit. Lwów 1895-1897 [La revue Ster sous la direction de Paulina Kuczalska-Reinschmit. Lvov 1895-1897], éditions de l'Université de Szczecin, 2017. 